\title{
Determination of generalized quadrangles with distinct elation points
}

\author{
Koen Thas
}

Received: 31 March 2005 / Accepted: 7 November 2005

(C) Springer Science + Business Media, LLC 2006

\begin{abstract}
In this paper, we classify the finite generalized quadrangles of order $(s, t), s, t>1$, which have a line $L$ of elation points, with the additional property that there is a line $M$ not meeting $L$ for which $\{L, M\}$ is regular. This is a first fundamental step towards the classification of those generalized quadrangles having a line of elation points.
\end{abstract}

Keywords Generalized quadrangle $\cdot$ Elation generalized quadrangle $\cdot$ Translation generalized quadrangle $\cdot$ Moufang condition $\cdot$ Symmetry $\cdot$ Regularity $\cdot$ Classification

Mathematics Subject Classification (2000): 51E12, 51E20, 20B25, $20 \mathrm{E} 42$

\section{Introduction and statement of the main result}

A (finite) generalized quadrangle $(G Q)$ of $\operatorname{order}(s, t)$ is an incidence structure $\mathcal{S}=$ $(P, B, I)$ in which $P$ and $B$ are disjoint (nonempty) sets of objects called points and lines respectively, and for which $I$ is a symmetric point-line incidence relation satisfying the following axioms.

(1) Each point is incident with $t+1$ lines $(t \geq 1)$ and two distinct points are incident with at most one line.

(2) Each line is incident with $s+1$ points $(s \geq 1)$ and two distinct lines are incident with at most one point.

(3) If $p$ is a point and $L$ is a line not incident with $p$, then there is a unique point-line pair $(q, M)$ such that pIMIqIL.

K. Thas $(\bowtie)$

Ghent University, Department of Pure Mathematics and Computer Algebra, Krijgslaan 281, S22,

B-9000 Ghent, Belgium

e-mail: kthas@cage.UGent.be 
If $s=t$, then $\mathcal{S}$ is also said to be of order $s$.

There is a point-line duality for GQ's of order $(s, t)$ for which in any definition or theorem the words "point" and "line" are interchanged and also the parameters. Normally, we assume without further notice that the dual of a given theorem or definition has also been given. Also, sometimes a line will be identified with the set of points incident with it without further notice. This will be done frequently.

For notation and definitions not explicitly mentioned here, we refer to the monograph Finite Generalized QuAdRANGLEs by S.E. Payne and J.A. Thas [25]. For an extensive survey on recent results on automorphisms and characterizations of GQ's, see [34].

Let $\mathcal{S}=(P, B, I)$ be a GQ of $\operatorname{order}(s, t), s, t>1$.

An elation about the point $p$ is either the identity, or a collineation of $\mathcal{S}$ that fixes $p$ linewise and no point of $P \backslash p^{\perp}$. By definition, the identity is an elation (about every point). If $p$ is a point of the GQ $\mathcal{S}$ for which there exists a group $G$ of elations about $p$ which acts regularly on the points of $P \backslash p^{\perp}$, then $\mathcal{S}$ is said to be an elation generalized quadrangle (EGQ) with elation point $p$ and elation group (or base-group) $\mathrm{G}$, and we often write $\left(\mathcal{S}^{(p)}, G\right)$ for $\mathcal{S}$, or $\left(\mathcal{S}^{p}, G\right)$.

The natural models of finite generalized quadrangles for which each point is an elation point are the so-called 'classical' and 'dual classical' examples, as defined by J. Tits in [10]. Those are constructed as follows.

(a) Consider a nonsingular quadric of Witt index 2, that is, of projective index 1 , in $\mathbf{P G}(3, q), \mathbf{P G}(4, q), \mathbf{P G}(5, q)$, respectively. The points and lines of the quadric form a generalized quadrangle which is denoted by $\mathcal{Q}(3, q), \mathcal{Q}(4, q), \mathcal{Q}(5, q)$, respectively, and has order $(q, 1),(q, q),\left(q, q^{2}\right)$, respectively.

(b) The points of $\mathbf{P G}(3, q)$ together with the totally isotropic lines with respect to a symplectic polarity form a GQ $W(q)$ of order $q$.

(c) Let $\mathcal{H}$ be a nonsingular Hermitian variety in PG(3, $\left.q^{2}\right)$, respectively $\mathbf{P G}\left(4, q^{2}\right)$. The points and lines of $\mathcal{H}$ form a generalized quadrangle $H\left(3, q^{2}\right)$, respectively $H\left(4, q^{2}\right)$, which has order $\left(q^{2}, q\right)$, respectively $\left(q^{2}, q^{3}\right)$.

Vice versa, using the ClassifiCATION OF Finite SiMPlE GROUPS (CFSG), see e.g. [9, 15], F. Buekenhout and H. Van Maldeghem were the first to obtain the converse [4]: if a finite generalized quadrangle has the property that each point is an elation point, then it is one of the classical or dual classical examples. Recently, the author of this paper and H. Van Maldeghem found a classification-free proof of that result. Now consider an EGQ $\mathcal{S}$ of order $(s, t), s \neq 1 \neq t$. Then, by transitivity, we clearly have the following possibilities for $\mathcal{S}$ :

(a) $\mathcal{S}$ has precisely one elation point;

(b) $\mathcal{S}$ has a line $L$ of elation points;

(c) each point of $\mathcal{S}$ is an elation point, and $\mathcal{S}$ is classical or dual classical.

In this paper, we will be concerned with the classification of GQ's of Type (b). Without additional hypotheses, this case seems completely hopeless at present, even if one allows the use of CFSG. In [37], such a classification was obtained with the following additional hypothesis:

(AB) There is a point on $L$ so that the corresponding elation group is abelian. 
If $p I L$ is such a point, then $p$ is called a translation point. If $(\mathrm{AB})$ is satisfied, all points incident with $L$ are translation points. The result we obtained was the following (for notions not explicitly defined yet, see Section 2, or [25] and [34]):

Theorem 1.1. (K. Thas [37]) Supposes $\mathcal{S}$ is a generalized quadrangle of order $(s, t), s \neq 1 \neq t$, with two distinct collinear translation points. Then we have one of the following:

(i) $s=t$ and $\mathcal{S} \cong \mathcal{Q}(4, s)$;

(ii) $t=s^{2}$, $s$ is even and $\mathcal{S} \cong \mathcal{Q}(5, s)$;

(iii) $t=s^{2}, s$ is odd, and $\mathcal{S}$ is the translation dual of the point-line dual of a flock $G Q$ $\mathcal{S}(\mathcal{F})$.

If a $G Q \mathcal{S}$ has two non-collinear translation points, then $\mathcal{S}$ is always of classical type, i.e. isomorphic to one of $\mathcal{Q}(4, s), \mathcal{Q}(5, s)$.

In [38], the converse of (iii) was (unexpectedly) obtained:

Theorem 1.2. (K. Thas [38]) The non-classical GQ's of order $(s, t)$, where $1<s<$ $t$, which have distinct translation points are precisely those $G Q$ 's $\mathcal{S}$ which are the translation dual of the point-line dual of a flock $G Q \mathcal{S}(\mathcal{F})$, where $\mathcal{F}$ is nonlinear.

Each of these examples has the following essential properties (which characterizes the examples by Theorem 1.2, see [38]):

(a) they have some line $L$ each point of which is an elation point (in fact, each of these points is a translation point);

(b) each line $M$ of the GQ which meets $L$ is a regular line (including $L$ ).

Therefore, we propose the (theoretically much more general) problem to classify the finite generalized quadrangles of $\operatorname{order}(s, t), s, t>1$, having a line $L$ of elation points, satisfying the following additional assumption:

(R) There exists a line $M$ not concurrent with $L$ so that $\{L, M\}$ is a regular pair of lines.

The following main result will be obtained:

Main Theorem. Suppose $\mathcal{S}$ is a generalized quadrangle of order $(s, t), s \neq 1 \neq t$, which has a line L each point of which is an elation point. Furthermore, suppose that Property $(R)$ is satisfied for $L$. Then we have one of the following:

(i) $s=t$ and $\mathcal{S} \cong \mathcal{Q}(4, s)$;

(ii) $t=s^{2}$, $s$ is even and $\mathcal{S} \cong \mathcal{Q}(5, s)$;

(iii) $\mathcal{S}$ is the translation dual of the point-line dual of a flock $G Q \mathcal{S}(\mathcal{F})$.

Conversely, each of the classes described in (i)-(ii)-(iii) satisfies the assumptions of the theorem.

We wish to obtain the main result in a "local-to-global" sense, such as in the spirit of [22] and [36, 37, 41], so that each step in the proof is as transparent as possible for applications in more general situations. As such, the main result will be merely 
a corollary of much more general observations, see especially Theorem 5.1 (Main Structure Theorem).

In an appendix, we will describe a direct, but less general, approach for the case $s=t$.

Remark 1.3. (Near polygons and spreads of symmetry) B. De Bruyn [5], see also [6], has developed a construction method for near polygons [27] from spreads of symmetry of generalized quadrangles, and new spreads of symmetry would yield new near polygons. Many new classes of near polygons were thus discovered. For generalized quadrangles of order $\left(s, s^{2}\right), s>1$, only one class of examples is known admitting spreads of symmetry, namely the classical example $\mathcal{Q}(5, q), q=s$, arising from a nonsingular elliptic quadric in $\mathbf{P G}(5, q)$ (and in that case there is a unique class of spreads of symmetry).

In [7], the authors started to investigate theoretically general classes of GQ's admitting a spread of symmetry. The following is taken from [7].

Theorem 1.4. (B. De Bruyn and K. Thas [7]) (i) Let $\mathcal{S}$ be a TGQ of order $(s, t), t>$ $s>1$, which admits a spread of symmetry. Then $\mathcal{S}$ is isomorphic to $\mathcal{Q}(5, s)$.

(ii) Let $\mathcal{S}$ be an EGQ of order $\left(s, s^{2}\right), s>1$ and $s$ even, which admits a spread of symmetry. Then $\mathcal{S}$ is isomorphic to $\mathcal{Q}(5, s)$.

In [40], the odd case for Theorem 1.4(ii) was then obtained:

Theorem 1.5. (K. Thas [40]) Suppose $\mathcal{S}$ is an elation generalized quadrangle of order $\left(s, s^{2}\right), s>1$ and $s$ odd, which has a spread of symmetry $\mathbf{T}$. Then $\mathcal{S} \cong \mathcal{Q}(5, s)$.

Each of the (abstract) GQ's considered in Theorem 1.4 and Theorem 1.5 satisfies the assumptions of the Main Theorem. (Theorem 1.4(i) does not follow from it, however. Theorem 1.5 does, in combination with Theorem 1.4, but its proof is used in the proof of the Main Theorem. Also, here we need more group theory than in the proof of Theorem 1.5; we will need the classification of finite projective planes of Lenz-Barlotti class III [17].)

\section{Finite generalized quadrangles: further theory}

\subsection{Further theory}

Let $\mathcal{S}=(P, B, I)$ be a (finite) generalized quadrangle of order $(s, t), s \neq 1 \neq t$. Then $|P|=(s+1)(s t+1)$ and $|B|=(t+1)(s t+1)$. Also, $s \leq t^{2}$ and, dually, $t \leq s^{2}$, and $s+t$ divides $s t(s+1)(t+1)$.

If $\mathcal{S}$ is a GQ, then by $\mathcal{S}^{D}$ we denote its point-line dual.

Let $p$ and $q$ be points of $\mathcal{S}$. If $p=q$ or if $p$ and $q$ are on the same line (and then they are called 'collinear'), then we write $p \sim q$. The same notation is used for lines (and in that case, we speak of 'concurrent lines'). For $p \in P$, put $p^{\perp}=\{q \in P \| q \sim p\}$. For a pair of distinct points $\{p, q\}$, the trace of $\{p, q\}$ is defined as $p^{\perp} \cap q^{\perp}$, and we denote Springer 
this set by $\{p, q\}^{\perp}$. Then $\left|\{p, q\}^{\perp}\right|=s+1$ or $t+1$, according as $p \sim q$ or $p \nsucc q$. More generally, if $A \subseteq P, A^{\perp}$ is defined by $A^{\perp}=\bigcap\left\{p^{\perp} \| p \in A\right\}$. For $p \neq q$, the span of the pair $\{p, q\}$ is $\operatorname{sp}(p, q)=\{p, q\}^{\perp \perp}=\left\{r \in P \| r \in s^{\perp}\right.$ for all $\left.s \in\{p, q\}^{\perp}\right\}$. We have that $\left|\{p, q\}^{\perp \perp}\right|=s+1$ or $\left|\{p, q\}^{\perp \perp}\right| \leq t+1$ according as $p \sim q$ or $p \nsucc q$. If $p \sim q \neq p$, or if $p \nsucc q$ and $\left|\{p, q\}^{\perp \perp}\right|=t+1$, we say that the pair $\{p, q\}$ is regular. The point $p$ is regular provided $\{p, q\}$ is regular for every $q \in P \backslash\{p\}$. Regularity for lines is defined dually. One easily proves that either $s=1$ or $t \leq s$ if $\mathcal{S}$ has a regular pair of non-collinear points.

If $(p, L)$ is a non-incident point-line pair of a GQ, then by $\operatorname{proj}_{p} L$, we denote the unique line of the GQ which is incident with $p$ and concurrent with $L$. Dually, one defines $\operatorname{proj}_{L} p$.

A subquadrangle, or also $s u b G Q, \mathcal{S}^{\prime}=\left(P^{\prime}, B^{\prime}, I^{\prime}\right)$ of a GQ $\mathcal{S}=(P, B, I)$ of order $(s, t), s, t>1$, is a GQ for which $P^{\prime} \subseteq P, B^{\prime} \subseteq B$, and where $I^{\prime}$ is the restriction of $I$ to $\left(P^{\prime} \times B^{\prime}\right) \cup\left(B^{\prime} \times P^{\prime}\right)$. A subGQ of order $(s, 1), s>1$, is sometimes called a grid $($ or $(s+1) \times(s+1)-$ grid $)$.

\subsection{Symmetry in generalized quadrangles}

A collineation or automorphism of a generalized quadrangle $\mathcal{S}=(P, B, I)$ is a permutation of $P \cup B$ which preserves $P, B$ and $I$. Here, $A u t(\mathcal{S})$ denotes the automorphism group of $\mathcal{S}$.

An axis of symmetry $L$ of $\mathcal{S}$ is a line for which there is a full group of size $s$ of collineations of $\mathcal{S}$ fixing $L^{\perp}$ elementwise. Dually, one defines a center of symmetry. If a GQ $\left(\mathcal{S}^{(p)}, G\right)$ is an EGQ with elation point $p$, and if each line incident with $p$ is an axis of symmetry, then we say that $\mathcal{S}$ is a translation generalized quadrangle (TGQ) with translation point $p$ and translation group (or base-group) $G$. In such a case, $G$ is uniquely defined; $G$ is generated by all symmetries about every line incident with $p$, and $G$ is the set of all elations about $p$, see 8.3.2 of [25]. That $p$ is indeed a translation point in the sense of Section 1 follows from Theorem 2.2 below.

Theorem 2.1. ([25], 8.3.1) Let $\mathcal{S}=(P, B, I)$ be a $G Q$ of order $(s, t), s, t>1$. Suppose each line through some point $p$ is an axis of symmetry, and let $G$ be the group generated by the symmetries about the lines through $p$. Then $G$ is elementary abelian and $\left(\mathcal{S}^{(p)}, G\right)$ is a $T G Q$.

Theorem 2.2. ([25], 8.2.3) Suppose $\left(\mathcal{S}^{(x)}, G\right)$ is an EGQ of order $(s, t), s \neq 1 \neq t$. Then $\left(\mathcal{S}^{(x)}, G\right)$ is a TGQ if and only if $G$ is an (elementary) abelian group.

Remark 2.3. (i) Each line of the GQ's $\mathcal{Q}(4, s)$ and $\mathcal{Q}(5, s)$ is an axis of symmetry.

(ii) Each known GQ (see Chapter 3 of [41] for a detailed account), except for $H\left(4, q^{2}\right.$ ) and $H\left(4, q^{2}\right)^{D}$, contains axes of symmetry or is constructed from a GQ with axes of symmetry (up to duality), see [41] for a classification of the possible configurations of axes of symmetry in GQ's.

Finally, the following result will be essential for the proof of the Main Result: 
Theorem 2.4. (I. Bloemen, J. A. Thas and H. Van Maldeghem [2]) Each EGQ of $\operatorname{order}(p, t), p, t>1$, with $p$ a prime, is isomorphic to $W(p), \mathcal{Q}(4, p)$ or $\mathcal{Q}(5, p)$.

\section{Split BN-pairs of rank 1}

A (group with a) split $B N$-pair of rank 1 is a permutation group $(Y, G)$, where $G$ acts on $Y$, which satisfies the following properties.

(BN1) $G$ acts 2-transitively on $Y$;

(BN2) for every $y \in Y$ the stabilizer of $y$ in $G$ has a normal subgroup (called a root group) which acts regularly on $Y \backslash\{y\}$.

If $Y$ is a finite set, then the split BN-pair of rank 1 also is called finite. The following theorem classifies all finite split BN-pairs of rank 1 without using CFSG, see [26] and [18].

Theorem 3.1. ([18, 26]) Suppose $(Y, G)$ is a group with a finite split BN-pair of rank 1 , and suppose $|Y|=s+1$, with $s<\infty$. Then $G$ must be one of the following (up to isomorphism):

(i) a sharply 2-transitive group on $Y$;

(ii) $\operatorname{PSL}(2, s)$;

(iii) the Ree group $\mathbf{R}(\sqrt[3]{s})$ with $\sqrt[3]{s}$ an odd power of 3;

(iv) the Suzuki group $\mathbf{S z}(\sqrt{s})$ with $\sqrt{s}$ an odd power of 2;

(v) the unitary group $\operatorname{PSU}\left(3, \sqrt[3]{s^{2}}\right)$,

each in its natural action of degree $s+1$.

Every root group has order $s$. In all of the cases except in Case (i), $s$ is a prime power. We have that $|\operatorname{PSL}(2, s)|=(s+1) s(s-1)$ or $(s+1) s(s-1) / 2$, according to whether $s$ is even or odd; in the other cases, we have that $|\mathbf{R}(\sqrt[3]{s})|=(s+1) s(\sqrt[3]{s}-$ $1),|\mathbf{S z}(\sqrt{z})|=(s+1) s(\sqrt{s}-1)$, and $\left|\mathbf{P S U}\left(3, \sqrt[3]{s^{2}}\right)\right|=\frac{(s+1) s\left(\sqrt[3]{s^{2}}-1\right)}{\operatorname{gcd}(3, \sqrt[3]{s}+1)}(\operatorname{gcd}(a, b)$ denotes the greatest common divisor of $a$ and $b ; a, b \in \mathbb{N}$ ).

\section{Proof of the main theorem}

STANDING HYPOTHESIS. In this section, unless otherwise explicitly mentioned, $\mathcal{S}=$ $(P, B, I)$ is a $G Q$ of order $(s, t), s, t>1$, and $L \in B$ is a line of elation points so that Property $(R)$ holds (w.r.t. M). We will also suppose that $s>2$, and refer to Chapter 6 of [25] for the case $s=2$.

NotATION. Let $p I L$. Then by $G_{p}$ we denote the elation group of size $s^{2} t$ of elations with center $p$ as defined to exist by the STANDING HYPOTHESIS.

First of all, we note that $L$ is a regular line. For, suppose $M^{\prime}$ is an arbitrary line of $B \backslash L^{\perp}$. If $M \sim M^{\prime}$, then there is some element of $G_{\text {proj }_{L}}\left(M \cap M^{\prime}\right)$ mapping $M$ onto $M^{\prime}$. So $\left\{L, M^{\prime}\right\}$ is also a regular pair of lines. Now suppose $M^{\prime} \nsucc M$. By [3], there is a set of lines $\left\{M_{0}=M, M_{1}, \ldots, M_{r}=M^{\prime}\right\}$, where $r \in \mathbb{N}$, so that each $M_{i}$ does not meet $L$, and so that $M_{i} \sim M_{i+1}$ for $i=0,1, \ldots, r-1$. It now readily follows 
that there is some collineation in $\operatorname{Aut}(\mathcal{S})$ mapping $M$ onto $M^{\prime}$, and hence $\left\{L, M^{\prime}\right\}$ is a regular line pair. So $L$ is regular. Note that, as $L$ is regular, we have that $s \leq t$. We distinguish some cases.

\section{The CASE $s$ Even}

First suppose $s$ to be even. Then as $L$ is regular, by [16], [8] and [33], each point incident with $L$ is a translation point. By Theorem 1.1, it thus follows that we have one of the following possibilities:

(a) $s=t$ and $\mathcal{S} \cong \mathcal{Q}(4, s)$;

(b) $t=s^{2}$ and $\mathcal{S} \cong \mathcal{Q}(5, s)$.

\section{THE CASE $s=t$}

Let $s$ be odd. Define the following incidence structure $\Pi_{L}$ :

- Lines. Are just the lines of $L^{\perp}$;

- Points. Are all spans of the form $\{V, W\}^{\perp \perp}$, where $V$ and $W$ are distinct lines in $L^{\perp} ;^{1}$

- Incidence. Is inversed inclusion.

Then by 1.3 .1 of [25], $\Pi_{L}$ is a projective plane of order $s$. Fix some point $x I L$, and consider a line $N \nsim L$. Then $\left(G_{x}\right)_{N}$ has size $s$. In $\Pi_{L},\left(G_{x}\right)_{N}$ fixes $x$ linewise, and the point corresponding to $\{L, N\}^{\perp}$. One notes that $\left(G_{x}\right)_{N}$ acts faithfully on $\Pi_{L}$. As $\left(G_{x}\right)_{N}$ has size $s$ as a collineation group of $\Pi_{L}$, the line $N^{\prime}=\operatorname{proj}_{x} N$ is an axis of $\left(G_{x}\right)_{N}$ (and in $\mathcal{S}, \operatorname{proj}_{N} x$ is linewise fixed by $\left.\left(G_{x}\right)_{N}\right)$. So $\Pi_{L}$ is $(x, q x)$-transitive for each point $x I L$, implying that $\Pi_{L}$ is a plane of Lenz-Barlotti class III. By [17], $\Pi_{L}$ is Desarguesian, and hence

$$
\Phi(N)=\Phi=\left\langle\left(G_{p}\right)_{N} \| p I L\right\rangle
$$

acts as $\mathbf{S L}(2, s)$ on $\Pi_{L} \cdot{ }^{2}$ Also, by the preceding considerations, we immediately have the following property:

- PROPERTY $(M)_{l}$. For each pIL, and each $L^{\prime} \nsucc L$, the group $\left(G_{p}\right)_{L^{\prime}}$ fixes $\operatorname{proj}_{L^{\prime}} p$ linewise.

Assume that $\{L, N\}^{\perp \perp}=\left\{L, N=N_{1}, N_{2}, \ldots, N_{s}\right\}$, and define

$$
\Phi\left(N_{i}\right)=\left\langle\left(G_{p}\right)_{N_{i}} \| p I L\right\rangle
$$

Let $i \neq j ; i, j \in\{1,2, \ldots, s\}$, and consider $\Phi_{i, j}=\Phi\left(N_{i}\right) \cap \phi\left(N_{j}\right)$. Let $\mathfrak{N}_{i}$ be the kernel of the action of $\Phi\left(N_{i}\right)$ on $X=\{L, N\}^{\perp}$. Then $\Phi_{i, j} /\left(\mathfrak{N}_{i} \cap \Phi_{i, j}\right)$ is a subgroup

\footnotetext{
${ }^{1}$ If $V \sim W$, we sometimes identify $\{V, W\}^{\perp \perp}$ with $V \cap W$.

2 In fact, this observation could also be applied for the problem considered in [22,36]. The proofs presented there use more elementary results, though.
} 
of $\Phi\left(N_{i}\right) / \mathfrak{N}_{i} \cong \operatorname{PSL}(2, s)$ of size at least $(s+1) s / 2$, with the additional property that $\operatorname{gcd}\left(\left|\Phi_{i, j} /\left(\mathfrak{N}_{i} \cap \Phi_{i, j}\right)\right|, s\right) \neq 1$.

Now recall Dickson's classification of the subgroups of $\operatorname{PSL}(2, q)$, with $q=p^{h}, p$ a prime (see [20, Hauptsatz 8.27 , p. 213]); we list the possible subgroups $H \leq$ PSL $(2, q)$, as follows:

(a) $H$ is an elementary abelian $p$-group;

(b) $H$ is a cyclic group of order $k$, where $k$ divides $\frac{q \pm 1}{r}$, where $r=\operatorname{gcd}(q-1,2)$;

(c) $H$ is a dihedral group of order $2 k$, where $k$ is as in (ii);

(d) $H$ is the alternating group $A_{4}$, where $p>2$ or $p=2$ and $h \equiv 0 \bmod 2$;

(e) $H$ is the symmetric group $S_{4}$, where $p^{2 h}-1 \equiv 0 \bmod 16$;

(f) $H$ is the alternating group $A_{5}$, where $p=5$ or $p^{2 h}-1 \equiv 0 \bmod 5$;

(g) $H$ is a semidirect product of an elementary abelian group of order $p^{m}$ with a cyclic group of order $k$, where $k$ divides $p^{m}-1$ and $p^{h}-1$;

(h) $H$ is a PSL $\left(2, p^{m}\right)$, where $m$ divides $h$, or a PGL $\left(2, p^{n}\right)$, where $2 n$ divides $h$.

We only have the following four possibilities for $\Phi_{i, j} /\left(\mathfrak{N}_{i} \cap \Phi_{i, j}\right)$ if $\Phi_{i, j} /\left(\mathfrak{N}_{i} \cap\right.$ $\left.\Phi_{i, j}\right) \neq \operatorname{PSL}(2, s)$ (for reasons of convenience, we will write $\Phi^{\prime}$ for $\Phi_{i, j} /\left(\mathfrak{N}_{i} \cap \Phi_{i, j}\right)$ ):

(1) $\Phi^{\prime}$ is a semidirect product of an elementary abelian group of order $p^{h}$ with a cyclic group of order $p^{h}-1$; in that case, $\Phi_{i, j}$ contains the Sylow $p$-subgroups of $\Phi\left(N_{i}\right)$, and hence coincides with $\Phi\left(N_{i}\right)$, contradiction;

(2) $\Phi^{\prime} \cong A_{4}$, then $\left|\Phi^{\prime}\right|=12$, and so $s=3$;

(3) $\Phi^{\prime} \cong A_{5}$, then $\left|\Phi^{\prime}\right|=60$, and so $s \leq 9$;

(4) $\Phi^{\prime} \cong S_{4}$, then $\left|\Phi^{\prime}\right|=24$, and so $s \leq 7$.

As $s$ is odd, we hence have that $s \in\{3,5,7,9\}$ if $\Phi^{\prime} \neq \mathbf{P S L}(2, s)$. But as $\mathcal{S}$ is an EGQ (for each point on [ $\infty]$ ), $\mathcal{S}$ is classical by Theorem 2.4 if $s \neq 9$, so $\mathcal{S}$ is isomorphic to one of $Q(4, s), W(s), s \in\{3,5,7\}$. As $\mathcal{S}$ contains a regular line, the dual of 3.3.1(i) of [25] yields that $\mathcal{S} \cong Q(4, s), s \in\{3,5,7\}$. If $s=9$, then by Theorem 6.3 of the Appendix, $\mathcal{S}$ is a TGQ, so that the result follows from [24] (see also the Appendix for more details).

Hence we infer that $\Phi_{i, j} /\left(\mathfrak{N}_{i} \cap \Phi_{i, j}\right)=\Phi\left(N_{i}\right) / \mathfrak{N}_{i}$, and it readily follows that each element of $\Phi\left(N_{i}\right)$ fixes $N_{j}$ (and thus also that each element of $\Phi\left(N_{i}\right)$ fixes $N_{i}$ ). So $\Phi\left(N_{i}\right)$ fixes all lines of $\{L, N\}^{\perp \perp}$.

Now fix an arbitrary $O \in\{L, N\}^{\perp}$, and define $H(O)=\left(G_{o}\right)_{N i}$, where $o=O \cap L$ and $i$ is arbitrary, and note that this definition is independent of $i$ by the preceding observations. Then $H(O)$ is a subgroup of $G_{o}$ of size $s$, fixing $O$ pointwise. Thus by Property $(\mathrm{M})_{l}$, we have that $H(O)$ fixes $O^{\perp}$ elementwise, and hence $O$ is an axis of symmetry. So each line of $L^{\perp}$ is regular (note that $\operatorname{Aut}(S)_{L}$ acts transitively on $L^{\perp} \backslash\{L\}$ ), and so each line of $\mathcal{S}$ is regular (as $\mathcal{S}$ is of order $s-$ see 1.3.6(iv) of [25]). By the dual of 5.2.1 of [25], $\mathcal{S} \cong \mathcal{Q}(4, s)$.

This concludes the proof of the main result for the case where $s=t$.

In fact, we can now obtain the following result:

Theorem 4.1. Let $\mathcal{S}$ be a GQ of order $s, s>1$ and $s \neq 9$, for which L is a regular line. Suppose $N \nsucc L$ is a line such that for each $n I N, \mathcal{S}$ admits a group of automorphisms fixing $\operatorname{proj}_{L}$ n linewise and acting regularly on $N \backslash\{n\}$. Then $\mathcal{S} \cong \mathcal{Q}(4, s)$. 
Proof: First suppose that $s>9$ and that $s$ is odd. By essentially the same arguments as in THE CASE $s=t$ (where one now defines $\Phi_{0, j}$ as $\left(\Phi\left(N_{0}\right)\right)_{N_{j}}$, with $N_{0}=N$ and $0 \neq j$, to conclude that $\left.\Phi_{0, j}=\Phi\right)$, it follows that each line of $\{L, N\}^{\perp}$ is an axis of symmetry, thus $\mathcal{S}$ is span-symmetric (in the terminology of Section 10.7 of [25]). All span-symmetric GQ's of order $s>1$ are classified, see [22], and, independently [36]; they are isomorphic to $\mathcal{Q}(4, s)$.

Now suppose that $s$ is even. With $\Phi_{0, j}$ as above, $\Phi_{0, j} /\left(\mathfrak{N}_{0} \cap \Phi_{0, j}\right)$ is a subgroup of $\Phi\left(N_{0}\right) / \mathfrak{N}_{0} \cong \mathbf{P S L}(2, s)$ of size at least $(s+1) s$, with the additional property that $\operatorname{gcd}\left(\left|\Phi_{0, j} /\left(\mathfrak{N}_{0} \cap \Phi_{0, j}\right)\right|, s\right) \neq 1$. Thus we may conclude the result if we are not in one of the cases (2)-(3)-(4). Now suppose we are. Then $s \leq 4$, and the theorem follows from 6.3 of [25] (recall that $s$ is a prime power).

Finally, suppose that $s \leq 8$; then $s \in\{3,5,7\}$, and $s$ is a prime. Fix a point $p I L$, and consider the group $H=\left(G_{p}\right)_{N}$ of size $s$. Then clearly, $H$ fixes all lines of $\{L, N\}^{\perp \perp}$, and thus $\operatorname{proj}_{p} N$ is an axis of symmetry. The result follows as before.

\section{A USEFUl OBSERVATION}

From now on, we suppose that $t>s$.

Let $N$ be, as before, non-concurrent with $L$. Put $\{L, N\}^{\perp}=X=\left\{M_{0}, M_{1}, \ldots, M_{s}\right\}$. For each $i \in\{0,1, \ldots, s\}$, put $y_{i}=M_{i} \cap L$ (so $L=\left\{y_{0}, y_{1}, \ldots, y_{s}\right\}$ ), and let $H_{i}=$ $\left(G_{y i}\right)_{N}$. Finally, put $n_{i}=M_{i} \cap N$ (so $N=\left\{n_{0}, n_{1}, \ldots, n_{s}\right\}$ ). We will sometimes write $\Omega$ for the set of points incident with the lines of $\{L, N\}^{\perp}$.

Let $\Lambda$ be an arbitrary $\Phi$-orbit in $\mathcal{S} \backslash \Omega$. Each $H_{i}, i=0,1, \ldots, s$, clearly fixes at least one line $O_{i}$ through $y_{i}$ different from $L$ and $M_{i}$, which is, as a point set, contained in $\Lambda$ (recall that $s$ is a prime power). Each point of $O_{i}$ is a point of $\Lambda \cup \Omega$, and hence the points on the lines of $\left\{O_{i}, L\right\}^{\perp}$ are completely contained in $\Lambda \cup \Omega$. Let $W$ be a line of $\left\{O_{i}, L\right\}^{\perp}$ which is not contained in $X$. The stabilizer $\Phi_{W}$ of $W$ in $\Phi$ acts transitively on $X \backslash\{Z\}$, where $Z \sim W$ and $Z \in X$. Hence each point of $L \backslash\{Z \cap W\}$ is incident with at least $s$ lines different from $L$, of which the point sets are completely contained in $\Lambda \cup \Omega$. As $\Phi$ acts transitively on the points of $L$, we thus obtain that $|\Phi| \geq|\Lambda| \geq s^{3}-s$. So, for each $M_{i}, M_{j} \in X, i \neq j$, and $U$ an arbitrary line through $y_{i}, M_{i} \neq U \neq L$, we have that

$$
\left|U^{\left(\Phi_{M_{i}}\right)_{M_{j}}}\right| \geq s-1 .
$$

Now suppose that $t<s^{2}$, and that there is a point $x I L$ and a point $y \nsucc x$ for which $\left|\{x, y\}^{\perp \perp}\right| \geq 3$. Then clearly, by transitivity, the latter holds for every point $x$ incident with $L$ and every $y \nsucc x$. So $\left|\left\{n_{i}, y_{j}\right\}^{\perp \perp}\right| \geq 3$. But then $(*)$ implies that

$$
\left|\left\{n_{i}, y_{j}\right\}^{\perp \perp}\right| \geq s-1,
$$

contradicting 1.4.1 of [25] and the fact that $s<t$.

If $t=s^{2}$, each span of non-collinear points has size 2 by 1.4.1 of [25].

We have obtained the following observation:

Observation 4.2. Suppose $\mathcal{S}$ satisfies the assumptions of the STANDING HYPOTHESIS. If $s<t$, then for each $x I L$, the elation group $G_{x}$ is the full set of such elations. 
Proof: The proof follows immediately from the fact that each span of non-collinear points which contains $x$ has size 2, in combination with 8.2.4 of [25].

\section{GENERALIZED QUADRANGLES OF ORDER $(s, t)$ WITH A REGULAR LINE OF ELATION POINTS, AND SPLIT BN-PAIRS OF RANK 1.}

We use the notation of the preceding paragraph.

Then note the following properties:

- $\Phi$ acts 2-transitively on $X$;

- for every $M_{i} \in X, H_{i}$ is a normal subgroup of $\Phi_{M_{i}}$ which acts regularly on $X \backslash\left\{M_{i}\right\}$; this normal subgroup is the group of elations about $x_{i}$ which acts regularly on $N \backslash\left\{n_{i}\right\}$, which is guaranteed to be unique by Observation 4.2.

Hence $(X, \Phi / \mathfrak{N})$ is a (finite) split BN-pair of rank 1 , where $\mathfrak{N}$ is the kernel of the action of $\Phi$ onto $X$, and $\Phi / \mathfrak{N}$ is one of the following groups as listed in Theorem 3.1 (recall that $s$ is odd):

(a) a sharply 2-transitive group;

(b) $\operatorname{PSL}(2, s)$;

(c) $\mathbf{R}(\sqrt[3]{s})$;

(d) $\operatorname{PSU}\left(3, \sqrt[3]{s^{2}}\right)$.

The Sharply 2-Transitive CASE, $s<t \leq s^{2}$

Suppose $\Phi$ acts sharply 2-transitively on $X$ (which has size $s+1$ ). Then by Theorem 3.4B (i) of [11], $s+1$ is a prime power. As $s$ is odd, $s+1$ is a power of 2, say $2^{m}$. Also, by [14], the fact that $\mathcal{S}$ is an EGQ and that $s \leq t$, implies that $s$ is the power of some (odd) prime $p$. Let us write $p^{n}+1=2^{m}$.

We distinguish two cases:

(a) $n=2 n^{\prime}$ IS EVEN. Then $\left(p^{n^{\prime}}+1\right)\left(p^{n^{\prime}}-1\right)+2=2^{m}$, a contradiction, as $p$ is odd.

(b) $n$ IS ODD. Then $p^{n^{\prime}}+1=(p+1)\left(p^{n-1}-p^{n-2}+\ldots+1\right)$, and this is only possible when $n=1$. So $\mathcal{S}$ is of order $(p, t)$, where $p$ is a prime. So by Theorem 2.4, $\mathcal{S}$ is classical or dual classical (a situation which can only occur when $s=3$, as $\Phi$ acts sharply 2-transitively on $X$ ).

Note that we have, in fact, obtained a proof of the following theorem:

Theorem 4.3. (i) Let $\mathcal{S}$ be an EGQ of order $(s, t), s, t>1$, where $s$ is the power of an odd prime. If $\mathcal{S}$ has some line $L$ so that the stabilizer of $L$ in the automorphism group of $\mathcal{S}$ contains a subgroup which acts sharply 2-transitively on $L$, then $s=3$, and $\mathcal{S}$ is isomorphic to $W(3), \mathcal{Q}(4,3)$ or $\mathcal{Q}(5,3)$.

(ii) Let $\mathcal{S}$ be an EGQ of order $(s, t), s, t>1$, where $s \leq t$ and $s$ is odd. If $\mathcal{S}$ has some line $L$ so that the stabilizer of $L$ in the automorphism group of $\mathcal{S}$ contains a subgroup which acts sharply 2-transitively on $L$, then $s=3$, and $\mathcal{S}$ is isomorphic to $W(3), \mathcal{Q}(4,3)$ or $\mathcal{Q}(5,3)$. 
THE CASES (B)-(C)-(D), $s<t \leq s^{2}$

In this paragraph, $(X, \Phi / \mathfrak{N})$ is a finite split $\mathrm{BN}$-pair of rank 1 , and we may assume that $\Phi / \mathfrak{N}$ is one of the following groups:

(b) $\operatorname{PSL}(2, s)$;

(c) $\mathbf{R}(\sqrt[3]{s})$

(d) $\operatorname{PSU}\left(3, \sqrt[3]{s^{2}}\right)$.

As $\mathfrak{N}$ leaves $X$ elementwise invariant, $\mathfrak{N}$ and the $H_{j}$ 's normalize each other. Also, for each $H_{i}$ we have that $H_{i} \cap \mathfrak{N}=\{\mathbf{1}\}$, thus, as the $H_{j}$ 's generate $\Phi$, we have that $\mathfrak{N}$ is in the center $Z(\Phi)$ of $\Phi$ (actually, $\mathfrak{N}$ is the center of $\Phi$ if we are not in Case (a), as $Z(\Phi / \mathfrak{N})=\{\mathbf{1}\})$. We now recall an argument due to W. M. Kantor, $\mathrm{cf}$. [22]. In each of the groups considered in (b)-(c)-(d), we have that, for arbitrary $i=0,1, \ldots, s, H_{i} \mathfrak{N} / \mathfrak{N} \cong H_{i}$ is contained in $\left(\Phi_{M_{i}} / \mathfrak{N}\right)^{\prime}$. As the actions of $\Phi_{M_{i}}$ on $H_{i}$ and $H_{i} \mathfrak{N} / \mathfrak{N}$ are equivalent, it follows that $H_{i} \leq \Phi^{\prime}$, so that $\Phi \leq \Phi^{\prime}$. So $\Phi$ is a perfect group, and $\Phi$ is a so-called perfect central extension of $\Phi / \mathfrak{N}$. Now by, e.g., [40], it follows that as $\Phi / \mathfrak{N}$ does not act sharply 2-transitively on $X, \Phi / \mathfrak{N} \cong \mathbf{P S L}(2, s)$.

Remark 4.4. (Alternative Argument) There is an alternative way to obtain the previous observation - that is, to obtain that $\Phi / \mathfrak{N} \cong \operatorname{PSL}(2, s)$. Fix an arbitrary point $p I L$, and consider $G_{p}$. Let $n I N$ be arbitrary but non-collinear with $p$, and let $L_{0}=$ $L, L_{1}, \ldots, L_{t}$, be the lines incident with $p$. For $i=0,1, \ldots, t$, define $N_{i}=\operatorname{proj}_{n} L_{i}$, and put $H_{i}=\left(G_{p}\right)_{N_{i}}$. Then since $L$ is an axis of symmetry, $H_{0}$ is a normal subgroup of $G_{p}$. By Theorem 2.1 (2.1.3) of [16], each element of $\left\{H_{1}, H_{2}, \ldots, H_{t}\right\}$ is an (elementary) abelian group. In particular, $\left(G_{p}\right)_{N}$ is abelian. Hence the split BN-pair $(X, \Phi / \mathfrak{N})$ has abelian root groups. The only such groups are given by:

(a) sharply 2-transitive groups;

(b) $\operatorname{PSL}(2, s)$.

By exactly the same argument as in THE CASE $s=t$, that is, by using Dickson's classification, we may now conclude that $\Phi$ fixes each line of $X^{\perp}$ (note that it is essential to identify $\Phi / \mathfrak{N}$ with $\operatorname{PSL}(2, s))$.

Assume that $\Phi$ acts semiregularly on $\mathcal{S} \backslash \Omega$. Let $\Lambda$ be an arbitrary $\Phi$-orbit in $\mathcal{S} \backslash \Omega$. As $|\Phi| \geq s^{3}-s$, and as $\Phi$ is a perfect central extension of $\Phi / \mathfrak{N} \cong \operatorname{PSL}(2, s)$, by $\left[15\right.$, p. 302] we may conclude that $|\Phi|=s^{3}-s$ and that $\Phi \cong \mathbf{S L}(2, s)$. Note that if $x \in \Lambda$, and if $M I x$ and $M \sim L$, that $M \backslash \operatorname{proj}_{L} x$ is completely contained in $\Lambda$ (cf. A USEFUl OBSERVATION). Since $\Lambda$ has size $s^{3}-s$, each point of $L$ is incident with precisely $s+1$ lines which are completely contained in $\Lambda \cup \Omega$ (as point sets). Define the following point-line incidence structure $\mathcal{S}^{\prime}=\left(P^{\prime}, B^{\prime}, I^{\prime}\right)$ :

- Lines. The elements of $B^{\prime}$ are the lines of $\mathcal{S}^{\prime}$ and they are of two types:

(1) the lines of $\{L, N\}^{\perp} \cup\{L, N\}^{\perp \perp}$;

(2) the lines of $\mathcal{S}$ which contain a point of $\Lambda$ and a point of $\Omega$.

- Points. The elements of $P^{\prime}$ are the points of the incidence structure and they are just the points of $\Omega \cup \Lambda$. 
- Incidence. Is induced by $I$.

Then by [40], $\mathcal{S}^{\prime}$ is a subGQ of $\mathcal{S}$ of order $s$. Thus, by 2.2.2(ii) of [25], $t=s^{2}$. Each of the elation points of $L$ in $\mathcal{S}$ is also an elation point of $\mathcal{S}^{\prime}$ (by a combination of 2.2.2 and 8.1.1 of [25]), and $L$ is a regular line in $\mathcal{S}^{\prime}$, so by THE CASE $s=t, \mathcal{S} \cong \mathcal{Q}(4, s)$. As there are $s+1$ distinct $\Phi$-orbits in $\mathcal{S} \backslash \Omega$, it immediately follows that $L$ is contained in at least (and then precisely, by straightforward counting) $s^{3}+s^{2}$ distinct subGQ's of $\mathcal{S}$ of order $s$, all isomorphic to $\mathcal{Q}(4, s)$. By [38] (see also, implicitly, [30]), it follows that each point incident with $L$ is a translation point, and so by [37], $\mathcal{S}$ is the translation dual of the point-line dual of a flock GQ $\mathcal{S}(\mathcal{F})$. Now suppose that $\Phi$ does not act semiregularly on $\mathcal{S} \backslash \Omega$. As $\Phi$ fixes $X^{\perp}$ elementwise, we then have that $\Phi$ contains a nonidentity element which fixes a subGQ $\mathcal{S}^{\prime}$ of $\mathcal{S}$ of order $s$ pointwise. Hence, by 2.2.2(ii) of [25] we can infer that $t=s^{2}$. Again, we have that $\mathcal{S}^{\prime} \cong \mathcal{Q}(4, s)$. If $\mathfrak{N}^{\prime}$ is the kernel of the action of $\Phi$ on $\mathcal{S}^{\prime}$, it follows that $\left|\mathfrak{N}^{\prime}\right|=2$ (by 1.4.2(ii) of [25]). As $\mathfrak{N}^{\prime}$ is a normal subgroup of $\Phi$ of size $2, \mathfrak{N}^{\prime} \leq Z(\Phi)$. On the other hand, if one considers the restriction of $\Phi$ to $\mathcal{S}^{\prime} \cong \mathcal{Q}(4, s)$, then it is straightforward to see that $\Phi / \mathfrak{N}^{\prime} \cong \mathbf{S L}(2, s)$ (as each of the $H_{i}$ 's induces a full group of symmetries about $M_{i}$ in $\mathcal{S}^{\prime}$, and recalling $[22,36])$. But this implies that $\Phi$ is a perfect central extension of $\mathbf{S L}(2, s)$ of size $2\left(s^{3}-s\right)$, which cannot occur (see p. 302 of [15]).

The main result follows.

\section{From elation points to centers of transitivity, and beyond}

It is our purpose to obtain the following result, that generalizes (but uses the proof of) the Main Result of [37], and Main Theorem 2 of [41]. The proof almost completely follows from the above, except for the sharply 2-transitive case. There, we have to use a group theoretical technique of [22]. As a corollary, we will show that in the main result, one can replace 'elation point' by 'center of transitivity'. In fact, we will even show that in each of the (local) subresults, one can replace 'regular action' by 'transitive action'.

Theorem 5.1. (Main Structure Theorem) Let $\mathcal{S}$ be a GQ of order $(s, t), s, t>1$ and $s \neq 9$, for which $L$ is a regular line. Suppose $N \ngtr L$ is a line such that for each $n I N$, $\mathcal{S}$ admits a group of automorphisms fixing $\operatorname{proj}_{L} n$ linewise and acting transitively on $N \backslash\{n\}$. Then one of the following holds:

(i) $\mathcal{S} \cong \mathcal{Q}(4, s)$;

(ii) $t=s^{2}$, and there are precisely $s+1$ subGQ's of order s, all isomorphic to $\mathcal{Q}(4, s)$, which mutually intersect in the $(s+1) \times(s+1)$-grid defined by $\{L, N\}^{\perp \perp}$.

Proof: First put $s=t$.

Start with assuming that $s$ is even. Write $s=p_{1}^{u_{1}} p_{2}^{u_{2}} \cdots p_{r}^{u_{r}}$, where $p_{1}, p_{2}, \ldots, p_{r}$ are distinct primes (one of them being 2), and where $u_{1}, u_{2}, \ldots, u_{r}$ are natural numbers. Let $G_{p}$ be the group of all whorls about $p$, that is, the group of collineations fixing $p$ linewise, where $p I L$ is arbitrary (but fixed). Let $i \in\{1,2, \ldots, r\}$, and consider a Sylow $p_{i}$-subgroup $H$ of $\left(G_{p}\right)_{N}$. In the projective plane $\Pi_{L}, H$ induces (faithfully) a collineation group fixing the point corresponding to $p$ linewise, and fixing the point 
corresponding to $\{L, N\}^{\perp}$. As $\operatorname{gcd}(|H|, s-1)=1, H$ fixes the line $\operatorname{proj}_{p} N$ (as a line of $\Pi_{L}$ ) pointwise. We conclude that $H$, as a collineation group of $\mathcal{S}$, fixes $\operatorname{proj}_{N} p$ linewise. Now consider the group

$$
H^{*}=\left\langle H^{\prime} \| H^{\prime} \quad \text { is a Sylow } p_{j} \text {-subgroup of }\left(G_{p}\right)_{N} \text { for some } j\right\rangle \text {. }
$$

Then $\left|H^{*}\right|$ is divisible by $s$, and $H^{*}$ is a group of whorls about both $p$ and $\operatorname{proj}_{N} p$. Suppose that $\left|H^{*}\right|>s$. Then by 8.1.1 of [25], there is a nontrivial element $\theta \in H^{*}$ fixing precisely a subGQ $\Gamma$ of $\mathcal{S}$ of order $(1, \mathrm{~s})$ elementwise. One notes that the order of $\theta$ divides $s-1$ (and hence that order is at least 3). Also, $\langle\theta\rangle$ acts semiregularly on the points of $\mathcal{S} \backslash \Gamma$. Now consider a line $U \in\{L, N\}^{\perp}$ which is not incident with a point of $\Gamma$. Then $\left|U^{\langle\theta\rangle}\right| \geq 3$, and each line of $U^{\langle\theta\rangle}$ is contained in $\{L, N\}^{\perp}$. Moreover, each line of $U^{\langle\theta\rangle}$, and hence of $\{L, N\}^{\perp}$, is concurrent with the same $s+1$ lines of $\Gamma$. This is a contradiction (for, in a GQ of order $(s, t), s, t>1$, a span of a regular pair of non-concurrent lines cannot have the property that its trace is contained in a subGQ of order $(1, t))$, so $\left|H^{*}\right|=s$, and it follows that $H^{*}$ is a normal subgroup of $\left(G_{p}\right)_{N}$. Hence with $\Phi$ the group generated by the $H^{*}$ 's (letting $p$ vary), and with $\mathfrak{N}$ the kernel of its action on $X,(X, \Phi / \mathfrak{N})$ is a split BN-pair of rank 1 .

Let $s$ be odd. Suppose $x I L$ is so that there is some point $y I N, y \nsucc x$ for which $\{x, y\}^{\perp \perp}$ has size at least 3. Then by A USEFUl OBSERVATION, we have that $\{x, y\}$ is a regular pair of points, and this is true for all $x I L$ and all $y I N, x \neq y$. Now applying the same deduction as in the even case, and with the same notation, we may conclude that $(X, \Phi / \mathfrak{N})$ is a split BN-pair of rank 1 (in fact, it will be clear later on that this case cannot occur).

Now suppose, for general $(s, t), 1 \neq s \leq t$, that each span of two non-collinear points of which one point is incident with $L$ and the other with $N$, has size 2 , and note that if $s<t$, we already knew that this is always the case (the case where $s$ is even has the same proof). Let $y_{i} I L$ be arbitrary. Consider the group $H\left(M_{i}, y_{i}\right)$ of all whorls about $y_{i}$ fixing $N$. Then by the proof of 8.1.1 of [25], we have that each element $\theta$ of $H\left(M_{i}, y_{i}\right)$ that fixes at least two distinct elements of $X \backslash\left\{M_{i}\right\}$, is the identity on $X$ (and then also on $\mathcal{S}$, as $\theta$ is a whorl about $y_{i}$, and by 2.2.2 and 2.4.1 of [25]). So $\left(X \backslash\left\{M_{i}\right\}, H\left(M_{i}, y_{i}\right)\right)$ is a Frobenius group, and the Theorem of Frobenius (cf. [11, p. 86]) implies that $H\left(M_{i}, y_{i}\right)$ has a unique normal subgroup $H_{i}$ acting regularly on $X \backslash\left\{M_{i}\right\}$ and it has size $s$ as an automorphism group of $\mathcal{S}$. Hence with $\Phi$ the group generated by the $H\left(M_{j}, y_{j}\right)$ 's and $\mathfrak{N}$ the kernel of the action of $\Phi$ on $X,(X, \Phi / \mathfrak{N})$ is a finite split BN-pair of rank 1.

By the preceding considerations, we can conclude that we have the following possibilities:

(a) $\Phi / \mathfrak{N}$ is a sharply 2 -transitive group on $X$;

(b) $\Phi / \mathfrak{N} \cong \operatorname{PSL}(2, s), t=s^{2}$ and we have the conclusion of Theorem 5.1, except when $s=4$ and $\Phi$ is a perfect central extension of $\mathbf{S L}(2,4)$ of size $2\left(s^{3}-s\right)=120$ (which gives no contradiction with p. 302 of [15]); then we have that $\mathcal{S} \cong \mathcal{Q}(5,4)$ by [29], as $\mathcal{S}$ contains a subGQ of order 4 isomorphic to $\mathcal{Q}(4,4)$ (by the end of Section 4);

(c) $\Phi / \mathfrak{N} \cong \operatorname{PSL}(2, s), t=s$ and we have the conclusion of Theorem 5.1. 
Regarding Case (a), we refer to [22].

We are ready to obtain:

Theorem 5.2. Suppose $\mathcal{S}$ is a generalized quadrangle of order $(s, t), s \neq 1 \neq t$, which has a line L each point of which is a center of transitivity. Furthermore, suppose that Property $(R)$ is satisfied for L. Then we have one of the following:

(i) $s=t$ and $\mathcal{S} \cong \mathcal{Q}(4, s)$;

(ii) $t=s^{2}$, $s$ is even and $\mathcal{S} \cong \mathcal{Q}(5, s)$;

(iii) $\mathcal{S}$ is the translation dual of the point-line dual of a flock $G Q \mathcal{S}(\mathcal{F})$.

Conversely, each of the classes described in (i)-(ii)-(iii) satisfies the assumptions of the theorem.

Proof: By Theorem 5.1, we only have to consider the case $s=t=9$ (the case $s=$ $9<t$ then follows from the proof of Theorem 5.1 and the proof of the Main Result). Let $s=t=9$. By the proof of the previous result, we know that we are in one of the following cases:

(a) for each $x I L$ and $y \nsucc x,\{x, y\}^{\perp \perp}=\{x, y\}$;

(b) each point incident with $L$ is regular.

Suppose we are in Case (a), and fix some point pIL. Then by 8.2.4 of [25], the group of all whorls about $p$ contains a normal subgroup which acts regularly on the points of $\mathcal{S}$ not collinear with $p$. Therefore $\mathcal{S}^{p}$ is an EGQ, and the result follows from the Appendix to this paper.

Suppose we are in Case (b). As $s=9$ is odd, 1.5.2(v) of [25] implies that $L$ is antiregular (cf. 1.3 of [25]). This contradicts the fact that $L$ is regular. The theorem is proved.

\section{Local moufang conditions and the main observations: classification}

J. Tits [32] defines a generalized quadrangle of Moufang type, or a Moufang generalized quadrangle, as a generalized quadrangle $\mathcal{S}=(P, B, I)$ in which the following conditions hold:

(M) for any dual panel $(p, L, q)$ of $\mathcal{S}$ (so $p I L I q \neq p$ ), the group of all automorphisms of $\mathcal{S}$ fixing $p$ and $q$ linewise and $L$ pointwise is transitive on the points which are incident with a given line $U I p, U \neq L$, and different from $p$;

$\left(\mathrm{M}^{\prime}\right)$ the point-line dual notion of $(\mathrm{M})$.

A GQ which satisfies either Property $(\mathbf{M})$ or Property $\left(\mathbf{M}^{\prime}\right)$ is generally called half Moufang.

The Moufang condition for generalized quadrangles is a special case of the Moufang condition for generalized polygons, as introduced by Tits in [44]. Generalized quadrangles are precisely the generalized 4-gons. One of the great subtheories in the theory of finite projective planes - i.e. the generalized 3-gons - is the Lenz-Barlotti classification $[23,1]$, which determines the possible subconfigurations of point-line 
pairs $(p, L)$ of a projective plane for which the plane is $(p, L)$-transitive. If $p I L$, then one also speaks of the Lenz classification of projective planes.

For GQ's, a Lenz classification would be a determination of the possible subconfigurations of triples $(p, L, q)$ of a GQ, where $p I L I q \neq p$, for which the GQ is Moufang. Some configurational results were already obtained by several authors, see [39] for a detailed account:

- the result of J.A. Thas, S.E. Payne and H. Van Maldeghem [32], which asserts that every half Moufang GQ is automatically Moufang;

- the result of P. Fong and G.M. Seitz [12, 13] for generalized quadrangles, which implies that each Moufang GQ is classical or dual classical ${ }^{3}$;

- the classification of K. Thas and H. Van Maldeghem [43] of the GQ's for which each point is an elation point (the so-called 'strong elation generalized quadrangles'), which is more general than the first result in this enumeration.

In [41], we then presented a theory which classified the possible subconfigurations

$$
\{(p, L, q) \| \quad(p, L, q) \text { is Moufang and } L \text { is regular }\}
$$

i.e. the possible subconfigurations of axes of symmetry of a GQ. That manuscript also contains the solutions of several open problems in the field.

The most general natural Moufang condition for GQ's $\mathcal{S}=(P, B, I)$, which was introduced in [43, 42], is the following:

(UM) for any panel $(p, L, q)$ of $\mathcal{S}$, and any line $M I q, M \neq L$, the group of all automorphisms of $\mathcal{S}$ fixing all lines incident with $p$ and fixing $M$, acts transitively on the set of points incident with $M$, and different from $q$;

$\left(\mathrm{UM}^{\prime}\right)$ The point-line dual notion of (UM).

The main result of the present paper, especially the formulation of Theorem 5.1, immediately translates into the following contribution to the problem:

Theorem 6.1. Let $\mathcal{S}$ be a $G Q$ of order $(s, t), s, t>1$ and $s \neq t$, for which $L$ is a regular line.

(i) Suppose $s \neq 9$, and let $N \nsucc L$ be a line such that for each $n I N,\left(\operatorname{proj}_{L} n, N, n\right)$ satisfies (UM). Then one of the following holds:

(a) $\mathcal{S} \cong \mathcal{Q}(4, s)$;

(b) $t=s^{2}$, and there are precisely $s+1$ subGQ's of order $s$, all isomorphic to $\mathcal{Q}(4, s)$, which mutually intersect in the $(s+1) \times(s+1)$-grid defined by $\{L, N\}^{\perp \perp}$.

(ii) Suppose that for all $N \nsim$ L and each $n I N,\left(\operatorname{proj}_{L} n, N, n\right)$ satisfies $(U M)$. Then one of the following holds:

\footnotetext{
${ }^{3}$ Later on, work of S.E. Payne and J.A. Thas culminated in an almost complete, elementary proof of that result, see Chapter 9 of [25]. Using slightly more group theory, first W.M. Kantor [21] and then the author [34] completed this geometric approach. Without any group theory, J.A. Thas recently obtained the same result [31]. We also refer to the work of J. Tits and R. Weiss [46].
} 
(a) $\mathcal{S} \cong \mathcal{Q}(4, s)$

(b) $t=s^{2}$, $s$ is even and $\mathcal{S} \cong \mathcal{Q}(5, s)$;

(c) $\mathcal{S}$ is the translation dual of the point-line dual of a flock $G Q \mathcal{S}(\mathcal{F})$.

The converse also holds.

Remark 6.2. If one replaces (UM) in Theorem 6.1 by (M), then it is not so hard to obtain the same conclusion of Theorem 6.1, only with the use of the classification of span-symmetric generalized quadrangles of order $s$, the main result of [37] and Main Theorem 2 of [41]. We leave the proof for the interested reader.

\section{Appendix A: Alternative Proof for the Case $s=t$}

In the appendix, we will obtain the Main Result for THE CASE $s=t$ as a corollary of the following theorem.

Theorem 6.3. Let $\left(\mathcal{S}^{x}, H\right)$ be an EGQ of order $s, s>1$, for which there is a regular line L incident with $x$. Then $\left(\mathcal{S}^{x}, H\right)$ is a $T G Q$.

Proof: If $s$ is even, see [33]. Suppose $s$ is odd. By [33], $H$ contains a (full) group of symmetries $H(L)$ of size $s$ about $L$. It is clear that $H(L)$ is a normal subgroup of $H$. Now consider the affine plane $\mathcal{A}_{L}$ which arises from $\Pi_{L}$ by deleting $L$ and the points incident with $L$. Then $\mathcal{A}_{L}$ is a translation plane (cf. [19, p. 100]) with translation group $H / H(L)$, hence $H / H(L)$ is (elementary) abelian. By Theorem 2.3 of [16], it follows that $H$ is abelian, and the theorem follows.

So, under the STANDING HYPOTHESIS of the main result (and using the same notation), it follows that each point on $L$ is a translation point, hence every line of $\mathcal{S}$ is regular. So $\mathcal{S} \cong \mathcal{Q}(4, s)$ by the point-line dual of 5.2.1 of [25], and by 3.2.1 of loc. cit.

We also have

Corollary 6.4. Let $\mathcal{S}=\left(\mathcal{S}^{x}, H\right)$ be an EGQ of order 9, for which there is a regular line L incident with $x$. Then $\mathcal{S} \cong \mathcal{Q}(4,9)$.

Proof: By Theorem $6.3,\left(\mathcal{S}^{x}, H\right)$ is a TGQ with translation point $x$. The corollary then follows from IX of [24].

Acknowledgements The author is a Postdoctoral Fellow of the Fund for Scientific Research - Flanders (Belgium).

\section{References}

1. A. Barlotti, "Sulle possibili configurazioni del sistema delle coppie punto-retta $(A, a)$ per cui un piano grafico risulta $(A, a)$-transitivo," Convegno internazionale: Reticoli e geometrie proiettive, Palermo, 2529 ottobre 1957, Messina, 30 ottobre 1957, Edito dalla Unione Matematica Italiana con il contributo del Consiglio Nazionale delle Ricerche Edizione Cremonese, Rome (1958), 75-78. 
2. I. Bloemen, J.A. Thas and H. Van Maldeghem, "Elation generalized quadrangles of order ( $p, t), p$ prime, are classical," J. Statist. Plann. Inference 56, Special issue on orthogonal arrays and affine designs, Part I (1996), 49-55.

3. A.E. Brouwer, "The complement of a geometric hyperplane in a generalized polygon is usually connected," Finite Geometry and Combinatorics (Deinze, 1992), London Math. Soc. Lecture Note Ser. 191, Cambridge Univ. Press, Cambridge (1993), 53-57.

4. F. Buekenhout and H. Van Maldeghem, "Finite distance-transitive generalized polygons," Geom. Dedicata 52 (1994), 41-51.

5. B. De Bruyn, "Generalized quadrangles with a spread of symmetry," European J. Combin. 20 (1999), 759-771.

6. B. De Bruyn, Recent Results on Near Polygons: A Survey, Generalized Polygons, Proceedings of the Academy Contact Forum 'Generalized Polygons', 20 October 2000, Palace of the Academies, Brussels, Edited by F. De Clerck et al. (2001), 39-60.

7. B. De Bruyn and K. Thas, "Generalized quadrangles with a spread of symmetry and near polygons," Illinois J. Math. 46 Fall (2002), 797-818.

8. X. Chen and D. Frohardt, "Normality in a Kantor family," J. Combin. Theory A 64 (1993), 130136.

9. J.H. Conway, R.T. Curtis, S.P. Norton, R.A. Parker and R.A. Wilson, Atlas of Finite Groups, Maximal Subgroups and Ordinary Characters for Simple Groups, (With computational assistance from J. G. Thackray), Oxford University Press, Eynsham, 1985.

10. P. Dembowski, Finite Geometries, Berlin/Heidelberg/New York, Springer, 1968.

11. J.D. Dixon and B. Mortimer, Permutation Groups, Graduate Texts in Mathematics 163, Springer-Verlag, New York/Berlin/Heidelberg, 1996.

12. P. Fong and G.M. Seitz, "Groups with a (B,N)-pair of rank 2, I," Invent. Math. 21 (1973), 157.

13. P. Fong and G.M. Seitz, "Groups with a (B,N)-pair of rank 2, II," Invent. Math. 24 (1974), 191239.

14. D. Frohardt, "Groups which produce generalized quadrangles," J. Combin. Theory A 48 (1988), 139145.

15. D. Gorenstein, Finite Simple Groups. An Introduction to Their Classification, University Series in Mathematics, Plenum Publishing Corp., New York, 1962.

16. D. Hachenberger, "On finite elation generalized quadrangles with symmetries," J. London Math. Soc. (2) 53 (1996), 397-406.

17. C. Hering and W.M. Kantor, "On the Lenz-Barlotti classification of projective planes," Arch. Math. (Basel) 22 (1971), 221-224.

18. C. Hering, W.M. Kantor and G.M. Seitz, "Finite groups with a split BN-pair of rank 1, I," J. Algebra 20 (1972), 435-475.

19. D.R. Hughes and F.C. Piper, Projective Planes, Graduate Texts in Mathematics 6, Springer-Verlag, New York/Berlin, 1973.

20. B. Huppert, Endliche Gruppen I, Springer-Verlag, Berlin, 1967.

21. W.M. Kantor, "Automorphism groups of some generalized quadrangles," Adv. Finite Geom. and Designs, Proceedings Third Isle of Thorns Conference on Finite Geometries and Designs, Brighton 1990, Edited by J. W. P. Hirschfeld et al., Oxford University Press, Oxford (1991), 251-256.

22. W.M. Kantor, "Note on span-symmetric generalized quadrangles," Adv. Geom. 2 (2002), 197-200.

23. H. Lenz, "Kleiner Desarguesscher Satz und Dualität in projektiven Ebenen," Jberr. Deutsch. Math. Verein. 57 (1954), 20-31.

24. S.E. Payne and J.A Thas, "Generalized quadrangles with symmetry. II," Simon Stevin 49 (1975/76), 81-103.

25. S.E. Payne and J.A. Thas, Finite Generalized Quadrangles, Research Notes in Mathematics 110, Pitman Advanced Publishing Program, Boston/London/Melbourne, 1984.

26. E. Shult, "On a class of doubly transitive groups," Illinois J. Math. 16 (1972), 434-455.

27. E.E. Shult and A. Yanushka, "Near $n$-gons and line systems," Geom. Dedicata 9 (1980), 1-72.

28. K. Tent, "Half-Moufang implies Moufang for generalized quadrangles," J. Reine Angew. Math. 566 (2004), 231-236.

29. J.A. Thas, "3-Regularity in generalized quadrangles of order ( $\left.s, s^{2}\right)$," Geom. Dedicata 17 (1984), 3336.

30. J.A. Thas, "Characterizations of translation generalized quadrangles," Des. Codes Cryptogr. 23 (2001), 249-257. 
31. J.A. Thas, "Generalized quadrangles and the theorem of Fong and Seitz on (B,N)-pairs," Preprint.

32. J.A. Thas, S.E. Payne and H. Van Maldeghem, "Half Moufang implies Moufang for finite generalized quadrangles," Invent. Math. 105 (1991), 153-156.

33. K. Thas, "On symmetries and translation generalized quadrangles," Developments in Mathematics $\mathbf{3}$, Proceedings of the Fourth Isle of Thorns Conference 'Finite Geometries', 16-21 July 2000, Edited by A. Blokhuis et al., Kluwer Academic Publishers (2001), 333-345.

34. K. Thas, Automorphisms and Characterizations of Finite Generalized Quadrangles, Generalized Polygons, Proceedings of the Academy Contact Forum 'Generalized Polygons', 20 October 2000, Palace of the Academies, Brussels, Edited by F. De Clerck et al. (2001), 111-172.

35. K. Thas, "A theorem concerning nets arising from generalized quadrangles with a regular point," Des. Codes Cryptogr. 25 (2002), 247-253.

36. K. Thas, "Classification of span-symmetric generalized quadrangles of order s," Adv. Geom. 2 (2002), $189-196$.

37. K. Thas, "The classification of generalized quadrangles with two translation points," Beiträge Algebra Geom. 43 (2002), 365-398.

38. K. Thas, "Translation generalized quadrangles for which the translation dual arises from a flock," Glasgow Math. J. 45(3) (2003), 457-474.

39. K. Thas, "Symmetry in generalized quadrangles," Des. Codes Cryptogr. 29, Proceedings of the Oberwolfach conference "Finite Geometries (2001)" (2003), 227-245.

40. K. Thas, "A characterization of the classical generalized quadrangle $\mathcal{Q}(5, q)$ and the nonexistence of certain near polygons," J. Combin. Theory A 103 (2003), 105-120.

41. K. Thas, Symmetry in Finite Generalized Quadrangles, Frontiers in Mathematics 1, Birk"auser - Verlag, Basel-Boston-Berlin, 2004.

42. K. Thas and H. Van Maldeghem, "Moufang-like conditions for generalized quadrangles and classification of all finite quasi-transitive generalized quadrangles," Discrete Math. 294 (Proceedings of the 2003 Kloster Irsee Conference) (2005), 203-217.

43. K. Thas and H. Van Maldeghem, "Geometrical characterizations of some Chevalley groups of rank 2," Trans. Amer. Math. Soc., To appear.

44. J. Tits, "Sur la trialité et certains groupes qui s'en déduisent," Inst. Hautes Etudes Sci. Publ. Math. 2 (1959), 13-60.

45. J. Tits, "Classification of buildings of spherical type and Moufang polygons: a survey," Coll. Intern. Teorie Combin. Acc. Naz. Lincei, Roma 1973, Atti dei convegni Lincei 17 (1976), 229-246.

46. J. Tits and R. Weiss, Moufang Polygons, Springer Monographs in Mathematics, Springer-Verlag, 2002. 